\title{
Eficácia de limpeza durante o beneficiamento do tomate de mesa
}

\author{
Effectiveness of cleaning fresh market tomatoes during post harvest handling
}

\author{
Ana Maria de Magalhães ${ }^{\mathrm{I}}$ Marcos David Ferreira ${ }^{\mathrm{II}}$ Celso Luiz Moretti ${ }^{\mathrm{III}}$
}

\section{RESUMO}

Neste trabalho avaliou-se a limpeza do tomate de mesa 'Débora' em relação à eficácia na qualidade dos frutos. Primeiramente, estudou-se a eficácia, com auxílio de esferas de borracha, variando-se as cerdas (náilon, sisal, PET e fibra de coco) e a rotação (40, 80, 120 e 160rpm) das escovas. Depois, com os melhores resultados, foram avaliadas a eficácia da limpeza em tomates e a qualidade pós-colheita dos frutos mensurada pela perda de massa (\%), atividade respiratória $\left(\mathrm{CO}_{2}\right)$ e evolução da concentração de etileno. No primeiro ensaio, as cerdas de náilon, sisal e fibra de coco combinadas com as altas rotações tornaram a limpeza mais eficaz. A cerda PET e a rotação de 40rpm foram ineficientes, apresentando um índice de limpeza (IL) inferior a 50\%. Para os tomates, o tratamento com náilon a 120rpm foi o mais eficaz na limpeza ( $I L=91 \%)$; todavia, o tratamento com náilon $160 \mathrm{rpm}$ apresentou um IL inferior (88\%), pois, devido à alta velocidade, os frutos pulavam e não eram envoltos pelas cerdas. Menores IL foram obtidos com fibra de coco a 80 e 120rpm, mas não estatisticamente diferentes. De modo geral, frutos limpos com náilon e sisal a 120rpm apresentaram as maiores alterações na qualidade pós-colheita quando comparados aos frutos limpos nessas mesmas cerdas a 160rpm. Maior flexibilidade das cerdas proporciona maior contato fruto/cerdas, com melhoria na eficiência de limpeza, o que pode provocar alterações na qualidade pós-colheita do fruto, porém não significativas.

Palavras-chave: Lycopersicon esculentum, pós-colheita, linha de beneficiamento, escovas, rotações.

\section{ABSTRACT}

This research evaluated the cleaning process of fresh market tomatoes Débora in relation to the efficiency cleaning and fruit quality. First, the efficiency cleaning was carried, with rubber spheres, changing the types of bristles (nylon, sisal fiber, PET and coconut fiber) as well as the rotation (40, 80, 120 and 160rpm) of rotary brushes. Afterwards, with the best results, the studies were carried out with fresh market tomatoes to evaluate cleaning efficiency and fruit quality measured by weight loss (\%), respiratory activity $\left(\mathrm{CO}_{2}\right)$ and ethylene evolution. In the first case, the nylon and sisal fiber were more efficient at higher rotations. PET bristles at 40rpm rotation were inefficient, presenting a cleaning index (CI) lower than 50\%. The nylon brush treatment at 120rpm was the most efficient cleaning process $(C I=91 \%)$. However, nylon brushes at $160 \mathrm{rpm}$ treatment showed the lowest CI (88\%). These results were probably associated to the fact that, at higher speeds, fruits tended to jump, causing a poor contact between bristles and fruits. The lowest CI was observed on coconut fiber at 80 and 120rpm treatments. In general, cleaned fruits with nylon and sisal fiber at 120rpm demonstrated higher post-harvest quality alterations when compared to cleaned fruits in these brushes at 160rpm. More bristles flexibility provides better contact fruit/bristles increasing cleaning efficiency, however, it can cause changes on post-harvest fruit quality, but no significant.

Key words: Lycopersicon esculentum, post harvest, packing line, brushes, rotation.

\section{INTRODUÇÃO}

O tomate é um produto de grande importância nutricional e econômica e seu consumo contribui para uma dieta saudável e bem equilibrada (NAIKA et al., 2006). No ano de 2005, a produção mundial de tomate foi superior a 122,5 milhões de toneladas, e o Brasil ficou em nono lugar com

IFaculdade de Engenharia Agrícola (FEAGRI), Universidade Estadual de Campinas (UNICAMP), Campinas, SP, Brasil.

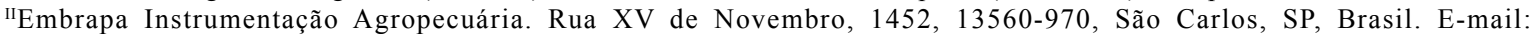
marcosferreira@cnpdia.embrapa.br. Autor para correspondência.

"IIEmbrapa Hortaliças, Rodovia Brasília/Anápolis, Brasília, DF, Brasil. 
aproximadamente 3,4 milhões de toneladas (FNP, 2006). No entanto, na comercialização de hortaliças, um fator desfavorável é o alto índice de perdas pós-colheita, que reduzem sensivelmente a disponibilidade desses produtos. No Brasil, essas perdas variam de $25 \%$ a $35 \%$ e começam no campo por ocasião da colheita, avançam durante o preparo dos produtos para comercialização e prosseguem nas centrais de abastecimento, nos atacadistas, nos varejistas e nos consumidores (VILELA et al., 2003).

A limpeza é realizada por razões sanitárias, pois muitos produtos recebem aplicação de defensivos agrícolas antes da colheita e muitos destes são de alta toxidade para o homem, mesmo em baixas concentrações. Assim, é necessária a remoção destes antes do embalamento (PELEG, 1985; ARTÉS \& ARTÉSHERNÁNDEZ, 2004). Nesse contexto, SIGRIST et al. (2002) definem que "a limpeza consiste na remoção de partículas de solo ou outros materiais estranhos da superfície das frutas e hortaliças por meio da lavagem do produto. Muitas vezes são utilizadas escovas para facilitar a operação".

Métodos de limpeza e desinfecção da superfície do produto usualmente envolvem tratamentos físicos e químicos, como a aplicação de água, o uso de detergentes, o uso de escovas e jatos de água (PARISH et al. 2003). Durante o processo de limpeza, em uma linha de beneficiamento, os frutos passam por uma série de escovas rotativas que transportam e friccionam os frutos, os quais são submetidos a jatos de água constantes (MILLER et al., 2001; ARTÉS \& ARTÉS-HERNANDEZ, 2004). Cerdas de diferentes origens, ou seja, de material sintético, vegetal ou animal, podem ser utilizadas nas escovas rotativas (PELEG, 1985), sendo elas, normalmente, de náilon, sisal, fibra de coco e pelo de cavalo (FRANCO et al., 2004).

Outro aspecto apontado por HYDE \& ZHANG (1992) é que as escovas utilizadas em uma linha de beneficiamento e classificação, de acordo com a sua finalidade, possuem diferentes velocidades. No entanto, cerdas rígidas, velocidade excessiva e/ou à excessiva exposição ao escovamento também podem causar danos e injúrias aos frutos, em especial, se as escovas utilizadas possuírem superfícies pontiagudas e rígidas (MILLER et al., 2001). SILVA et al. (2006) observaram que a eficiência de limpeza não está diretamente relacionada com o volume de água utilizado, mas sim à pressão da água, associada ao tempo de permanência dos frutos e à rotação das escovas. Vazões de $8,3 \times 10^{-6}$ e $13,3 \times 10^{-6} \mathrm{~m}^{3} \mathrm{~s}^{-1}$ apresentaram maiores índices de eficiência de limpeza, e para o tempo de permanência de $1 \mathrm{~min}$ a vazão de $13,3 \times 10^{-6} \mathrm{~m}^{3} \mathrm{~s}^{-1}$ apresentou maior eficiência de limpeza. As principais causas das perdas pós-colheita são as injúrias mecânicas, que se originam de forças externas de vibração, compressão e impacto (KAYS, 1991), que podem causar diferentes tipos de lesões, como abrasões, cortes, rupturas, amassamentos e injúrias internas (SARGENT et al., 1989; CHITARRA \& CHITARRA, 2005).

A perda de massa pode ser considerada a causa principal da deterioração na pós-colheita porque não resulta somente em perdas quantitativas (perda do peso vendável), mas também em perdas de aparência, textura e qualidade nutricional (KADER, 1992). ANDRADE JÙNIOR (1999) cita que, para o tomate de mesa, perdas de massa entre 3 e $6 \%$ são suficientes para a depreciação do produto. Semelhantes resultados foram observados por FERREIRA et al. (2006). KADER \& SALTVEIT (2003) relatam que, em geral, existe uma relação inversamente proporcional entre a atividade respiratória e vida pós-colheita de produtos agrícolas. Alta atividade respiratória está ligada a uma vida pós-colheita mais curta. Em produtos danificados, ocorre um aumento marcante na liberação de $\mathrm{CO}_{2}$ e captação de $\mathrm{O}_{2}$. As células mais próximas do ponto injuriado, que não foram mortas no processo, respiram muito mais rapidamente (LANA \& FINGER, 2000). KADER (1992) relata que incremento na taxa de concentração de etileno também se relaciona à incidência de injúrias físicas, dentre outros fatores.

O objetivo deste trabalho foi identificar quais são os parâmetros, o tipo de cerda e a rotação das escovas que tornam o processo de limpeza do tomate de mesa da cultivar 'Débora' mais eficiente, não afetando a qualidade pós-colheita dos frutos.

\section{MATERIAL E MÉTODOS}

Os ensaios foram realizados em laboratório, na Faculdade de Engenharia Agrícola, Universidade Estadual de Campinas (UNICAMP), utilizando-se o protótipo desenvolvido para testes na etapa de limpeza para o projeto UNIMAC - Unidade Móvel de Auxílio à Colheita. O equipamento é composto por dois módulos com $0,75 \mathrm{~m}$ de comprimento, $0,50 \mathrm{~m}$ de largura e cinco escovas transversais com $110 \mathrm{~mm}$ de diâmetro. No primeiro módulo, há um sistema de aspersão de água, que é formado por um bico aspersor com jato do tipo cone cheio (Spray Systems S.A., modelo Quick Full Jet-ProMax - QPHA-1.5). Em todos os ensaios, a vazão de água utilizada no processo de limpeza foi de $13,3 \mathrm{x}$ $10^{-6} \mathrm{~m}^{3} \mathrm{~s}^{-1}$, e o tempo de permanência das esferas/frutos no protótipo foi de $1 \mathrm{~min}$ (SILVA et al., 2006) 
Para se ter um parâmetro que caracterizasse o tipo de material utilizado nas cerdas das escovas, realizou-se um teste de flexibilidade das cerdas baseado nos estudos de RAWL et al. (1990). Para isso, cada cerda, com comprimento padronizado de $25 \mathrm{~mm}$, foi engastada em uma das extremidades na posição horizontal, e uma força peso $(\mathrm{P})$ vertical de $0,04 \mathrm{~N}$ foi aplicada sobre ela na outra extremidade. A flexibilidade de cada cerda foi obtida pelo índice de flexibilidade $(\mathrm{G})$, que é a relação entre a força peso (P) aplicada e a flexão da cerda (d).

$\mathrm{Na}$ primeira etapa do trabalho, foram avaliadas quatro tipos de cerdas (náilon com $0,15 \mathrm{~mm}$, sisal com $0,10 \mathrm{~mm}$, Tereftalato de polietileno (PET) com $0,15 \mathrm{~mm}$ e fibra de coco com 0,10 a $0,30 \mathrm{~mm}$ ) e quatro rotações (40, 80, 120 e 160rpm). Para isso, utilizaram-se esferas de borracha com $70 \mathrm{~mm}$ de diâmetro e $235 \mathrm{~g}$ de massa, que simulavam frutos de tomate, nas quais foi aplicada uma sujeira artificial sintética, preparada por meio da homogeneização de $20 \mathrm{~mL}$ de polietileno de alta densidade a 22,7\% (Meghwax EPE 350N - Megh), $20 \mathrm{~mL}$ de têmpera guache preta (Acrilex) e $6 \mathrm{~g}$ de carvão vegetal moído e peneirado, Tyler Mesh 48 (FRANCO et al., 2005).

Para avaliar a eficácia da limpeza, após passarem pelo protótipo, as esferas foram lavadas individualmente com $500 \mathrm{~mL}$ de água destilada, da qual se retirou amostras para a análise de turbidez. As análises foram realizadas em turbidímetro de bancada microprocessado (MS Tecnopon Instrumentação Científica, modelo TB 1000), com leitura de 0 a 100UNT e precisão de $2 \%$. Um índice de sujeira padrão $\left(\mathrm{UNT}_{\text {padrão }}\right)$ foi determinado por meio da análise de turbidez de 10 esferas totalmente sujas, ou seja, que não passaram pelo protótipo. Os resultados foram dados em função do índice de limpeza, descrito na equação 1 , que varia de $0 \%=$ sujo a $100 \%=$ limpo.

$$
\mathrm{IL}_{\mathrm{t}}=\left(\frac{\mathrm{UNT}_{\text {padrão }}-\mathrm{UNT}_{\text {amostra }}}{\mathrm{UNT}_{\text {padrão }}}\right) \times 100(\text { equação, } 1)
$$

Nessa equação, IL é igual a índice de limpeza obtido com o turbidímetro, \%; $\mathrm{UNT}_{\text {padrão }}$ í igual a valor médio da turbidez (UNT) para esferas $100 \%$ sujas; $\mathrm{UNT}_{\text {amostra }}$ é igual a valor de turbidez de cada amostra (esferas que passaram pelo processo de limpeza).

Na segunda etapa, foram utilizados tomates da cultivar 'Débora' com maturação verde maduro, colhidos manualmente na região de Mogi Guaçu, São Paulo, que foram submetidos à limpeza nos tratamentos que apresentaram uma melhor eficácia no processo de limpeza das esferas, ou seja, um índice de limpeza superior a $50 \%$.
Para mensurar a eficácia do processo de limpeza nos tomates, assim como foi feito com as esferas de borracha, após passarem pela etapa de limpeza, os frutos foram lavados individualmente com $500 \mathrm{~mL}$ de água destilada, da qual foram retiradas amostras para a análise de turbidez. O índice de sujeira padrão (UNT $\mathrm{Udrão}_{\text {pro }}$ utilizado foi referente à análise de turbidez de 10 frutos de tomate com a sujeira inicial de campo, ou seja, que não passaram pelo protótipo. Os resultados foram dados em função do índice de limpeza, descrito na equação 1 , que varia de $0 \%=$ sujo a $100 \%=$ limpo.

A qualidade dos frutos de tomate após a limpeza foi avaliada por meio da perda de massa (\%), atividade respiratória expressa em concentração de $\mathrm{CO}_{2}$ e evolução da concentração de etileno. A perda de massa foi determinada em relação à massa inicial dos frutos e dada em porcentagem. As pesagens foram realizadas após zero, um, três, cinco e sete dias de armazenamento, em balança digital Marte mod. AS1000C), com capacidade máxima de $1000 \mathrm{~g}$ e precisão de $0,1 \mathrm{~g}$.

Para a determinação da atividade respiratória e a evolução de etileno, aproximadamente $1 \mathrm{~kg}$ de tomates passados pelo processo de limpeza foi colocado em um frasco de vidro de $3,5 \mathrm{~L}$, hermeticamente fechado e armazenado a $24 \pm 1^{\circ} \mathrm{C}$ e $85 \pm 5 \%$ UR durante quatro horas. Com seringa própria para cromatografia, retirou-se $1 \mathrm{~mL}$ de gás da atmosfera interna do frasco, por meio de um septo de silicone adaptado à tampa deste (KAYS, 1991). As determinações das concentrações dos gases foram realizadas em cromatógrafo a gás (VARIAN modelo CG 3400). A atividade respiratória foi obtida pela diferença entre a concentração de $\mathrm{CO}_{2}$ durante as quatro horas e a concentração inicial. Os resultados foram expressos em mg CO $\mathrm{kg}^{-1} \mathrm{~h}^{-1}$. A evolução de etileno foi expressa em $\mu \mathrm{L} \mathrm{C}_{2} \mathrm{H}_{4} \mathrm{~kg}^{-1} \mathrm{~h}^{-1}$.

O delineamento experimental utilizado foi o inteiramente casualizado, em esquema fatorial e com três repetições $(n=10)$. Para análise dos resultados, utilizou-se a análise de variância, e a comparação das médias foi realizada pelo teste de Tukey $(\mathrm{P}<0,05)$, com o auxílio do programa Statgraphics plus 4.1.

\section{RESULTADOS E DISCUSSÃO}

No ensaio de flexibilidade das cerdas, observou-se que a cerda de náilon é a mais flexível, uma vez que apresentou o menor valor para o índice de flexibilidade $\left(\mathrm{G}_{\mathrm{c}}=68,34 \mathrm{~N} \mathrm{~m}^{-1}\right)$, e esse valor não diferiu significativamente $(\mathrm{P}>0,05)$ do valor obtido $\left(70,27 \mathrm{~N} \mathrm{~m}^{-1}\right)$ para a cerda de sisal. Todavia, esses dois valores diferiram $(\mathrm{P}<0,05)$ dos valores encontrados para a cerda 
de PET e fibra de coco, que diferiram também $(\mathrm{P}<0,05)$ entre si, com valores médios de 135,94 e 93,36 $\mathrm{N} \mathrm{m}^{-1}$, respectivamente.

No ensaio com as esferas de borracha, a limpeza foi mais eficaz com a cerda de náilon e rotação de 160rpm, que apresentou um índice de limpeza (IL) estatisticamente diferente $(\mathrm{P}<0,05)$ de praticamente todos os outros tratamentos, com exceção dos valores obtidos para os tratamentos sisal 160rpm, fibra de coco 120rpm e fibra de coco 160rpm (Figura 1). O aumento nas rotações de 40 para 160rpm elevou a eficácia de limpeza das esferas para todas as escovas. Segundo MILLER et al. (2001), isso ocorre porque o excessivo tempo de escovação e/ou a velocidade excessiva resultam em uma limpeza mais eficaz. Para PELEG(1985), a velocidade de rotação para ser satisfatória deve variar de 150 a 200rpm. Por sua vez, neste trabalho, as rotações de 80 e 120rpm, apesar de apresentarem menores valores que a rotação de $160 \mathrm{rpm}$, mostraram-se eficientes no processo de limpeza das esferas de borracha, com valores de IL superiores a 50\%, diferentemente do que ocorreu para a rotação de 40rpm. Nas rotações de 40, 80 e 160rpm, a eficácia no processo de limpeza foi maior para o tratamento utilizando a escova com cerdas de náilon, com valores médios para IL de 50; 58 e 80\%, respectivamente. Para a rotação de $120 \mathrm{rpm}$, o maior IL (78\%) foi obtido para o processo que utilizou a escova com cerdas de fibra de coco. De um modo geral, o IL obtido para a escova com cerdas PET foi muito baixo, com valores que variaram de 35 a $47 \%$, demonstrando sua ineficiência no processo de limpeza. Observa-se que as cerdas mais flexíveis, como as de náilon que apresentaram um $\mathrm{G}_{\mathrm{c}}$ de 68,34 , proporcionaram uma melhor eficácia de limpeza, quando comparadas às cerdas de PET, de maior rigidez $\left(\mathrm{G}_{\mathrm{c}}\right.$ de $\left.135,94 \mathrm{~N} \mathrm{~m}^{-1}\right)$. BRECHT (2002) e ARTÉS \& ARTÉS-HERNANDEZ (2004) colaboram com essa afirmação, indicando que, para uma limpeza eficaz, devem ser utilizadas cerdas mais macias e flexíveis.

Para os frutos de tomate, o tratamento com cerdas de náilon e rotação 120rpm foi o mais eficaz no processo de limpeza, com IL médio de $91 \%$, que diferiu estatisticamente $(\mathrm{P}<0,05)$ dos valores obtidos nos tratamentos com náilon a 80rpm, fibra de coco a 80rpm ou 120rpm (Figura 2). No processo de limpeza com as cerdas de fibra de coco, o IL aumentou com o aumento da rotação, diferentemente do que ocorreu com os frutos limpos com as escovas de náilon e sisal, em que a rotação de $120 \mathrm{rpm}$ apresentou-se mais eficiente que a de 160rpm. Uma justificativa para esse resultado é a de que, na rotação de 160rpm, devido à alta velocidade, os frutos pulavam e não eram envoltos pelas cerdas de maior flexibilidade (náilon e sisal). Esses resultados diferem do que indica PELEG (1985), o qual sugere, para melhor eficácia do processo de limpeza dos frutos, o uso de altas rotações, entre 150 e 200rpm. No entanto, esses resultados estão de acordo com o que sugerem MILLER \& ISMAIL (2005), que recomendam, para a limpeza de citros, a velocidade máxima de $120 \mathrm{rpm}$. No caso das cerdas de fibra de coco, por apresentarem

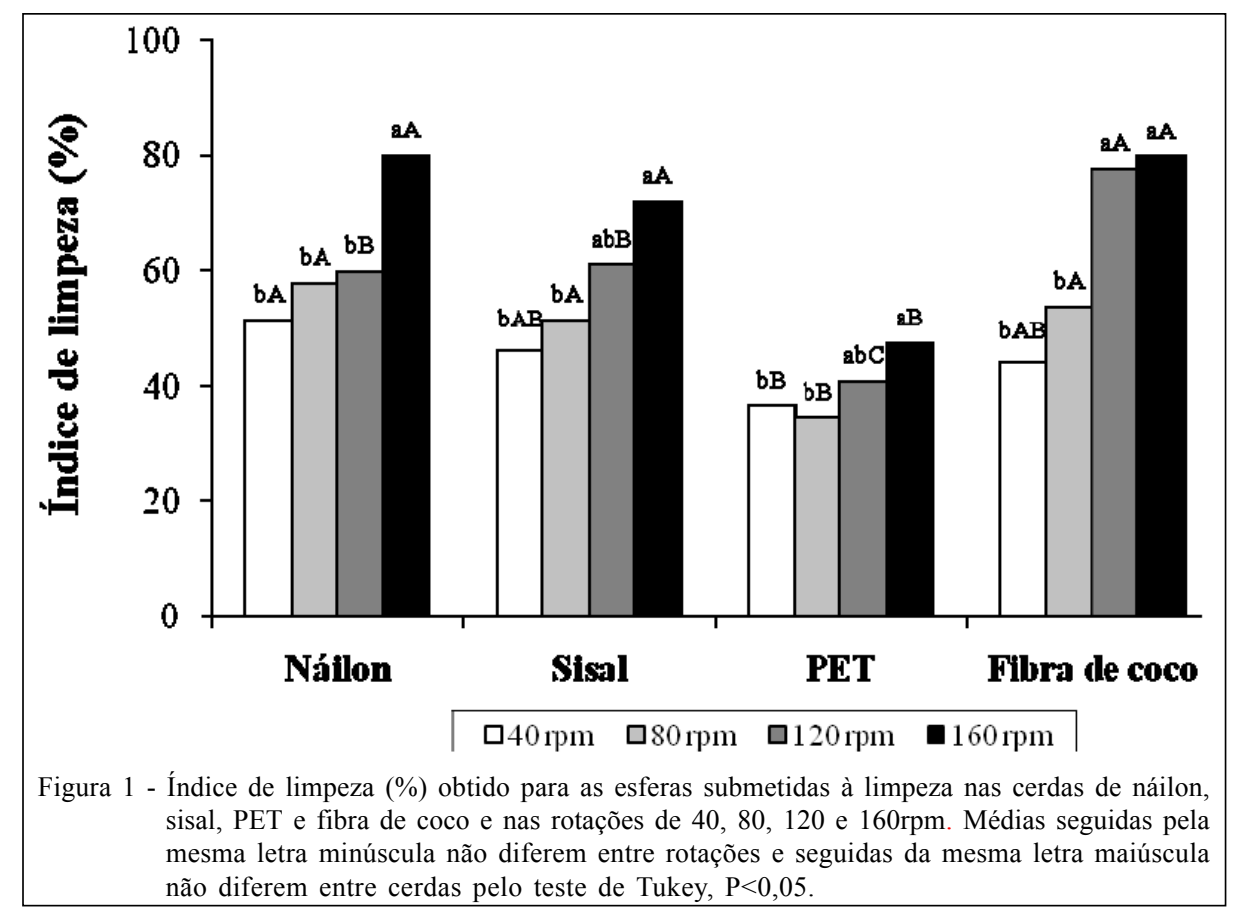

Ciência Rural, v.39, n.9, dez, 2009. 


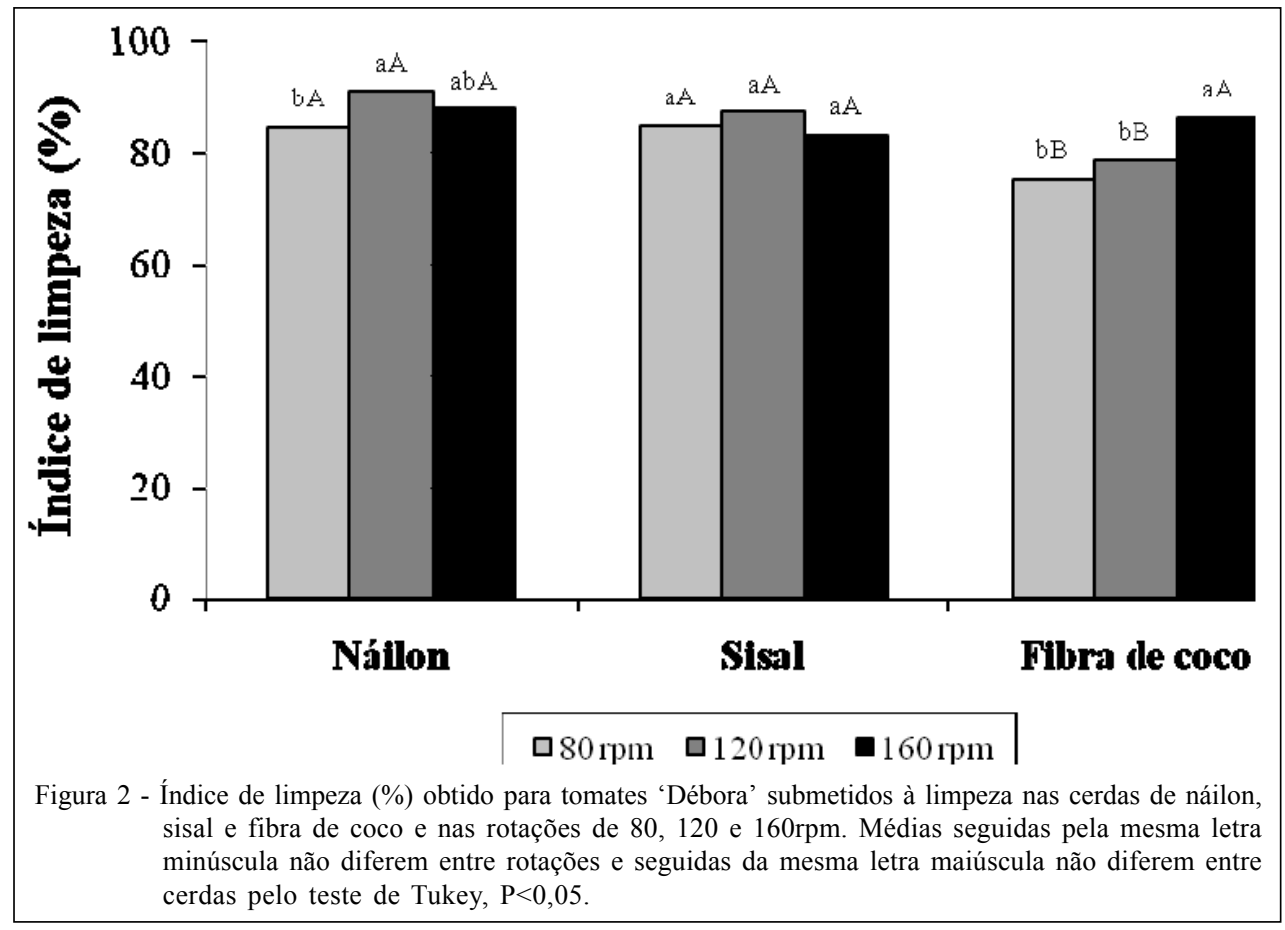

maior rigidez $\left(93,36 \mathrm{~N} \mathrm{~m}^{-1}\right)$, a limpeza era realizada por meio do contato pontual entre as cerdas e os frutos, que tendia a ser mais eficiente com o aumento da velocidade das rotações.

A perda de massa dos frutos foi crescente com o tempo de armazenamento, e os frutos de controle apresentaram os menores valores médios durante o armazenamento, com uma perda de massa que variou de $0,53 \%$ no primeiro a $3,41 \%$ no último dia (Tabela 1 ).
No primeiro dia, após o processo de limpeza, observouse que os frutos que foram limpos com as cerdas de náilon e sisal, que são mais flexíveis, e nas rotações de 80 e $120 \mathrm{rpm}$ apresentaram as maiores médias para a perda de massa, quando comparados aos frutos submetidos a essas mesmas cerdas, mas na rotação de 160rpm. Uma justificativa para esse resultado é a de que, na rotação de $160 \mathrm{rpm}$, devido à alta velocidade, os frutos pulavam durante o processo de limpeza e não

Tabela 1 - Valores médios da perda de massa (\%) obtida para os frutos submetidos à limpeza nas escovas com cerdas de náilon, sisal e fibra de coco e nas rotações de 80,120 e $160 \mathrm{rpm}$, nos dias um, três, cinco e sete.

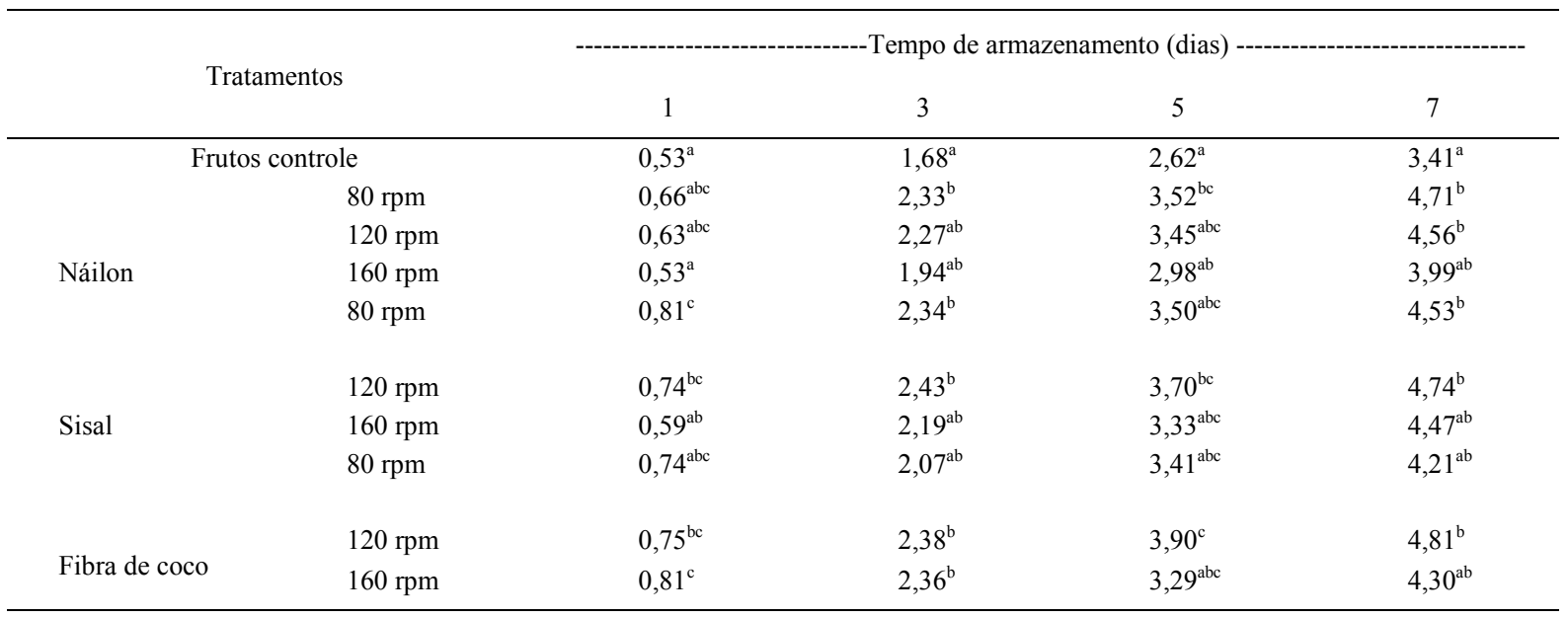

Médias com mesma letra na mesma coluna não diferem entre si pelo teste de Tukey,P $<0,05$.

Ciência Rural, v.39, n.9, dez, 2009. 
eram envoltos pelas cerdas, sendo um indicativo de que o envolvimento dos frutos pelas cerdas torna o processo de limpeza mais eficiente. Todavia, podem provocar a remoção da cera natural, que reveste o tomate (PELEG, 1985; BRECHT, 2002; PARISH et al., 2003), aumentando a perda de massa e alterando assim a qualidade do fruto logo após o processo de limpeza. As cerdas de fibra de coco, quando combinadas com as três rotações, provocaram elevados valores de perda de massa durante os sete dias de armazenamento, indicando que a rigidez das cerdas $\left(\mathrm{G}_{\mathrm{c}}=93,36 \mathrm{~N} \mathrm{~m}^{-1}\right)$ causou danos aos frutos (MILLER et al., 2001). Com o decorrer do armazenamento, observou-se que os frutos que foram submetidos ao processo de limpeza apresentaram valores semelhantes entre si para a perda de massa e superiores aos valores obtidos para os frutos de controle, demonstrando que o processo de limpeza provocou alterações na qualidade dos frutos. FERREIRA et al. (2004), também observaram que frutos de tomates 'Débora', submetidos ao manuseio, apresentam perda de massa significativamente superior aos frutos que não foram manipulados.

A atividade respiratória (AR) foi decrescente com o tempo em que os frascos permaneceram fechados para quase todos os tratamentos, sendo diferente somente no tratamento sisal 160rpm, que aumentou de 59,65 para $62,29 \mathrm{mg} \mathrm{CO}_{2} \mathrm{~kg}^{-1} \mathrm{~h}^{-1}$ da primeira para a segunda hora após a limpeza (Tabela 2). KADER \& MORRIS (1978) também observaram uma queda na AR do tomate. Segundo esses autores, uma elevada
AR inicial está relacionada a danos mecânicos durante a colheita e ao manuseio pós-colheita dos frutos. De um modo geral, a AR apresentada pelos frutos na primeira hora após o processo de limpeza foi superior aos valores encontrados por MOURA et al. (2005) e KADER \& MORRIS (1978). Altos valores de AR iniciais indicam que o processo de limpeza provocou alterações no metabolismo dos frutos. CALEGARIO et al. (2001) observaram que o manuseio pós-colheita influenciou o aumento da AR do tomate, pois frutos de tomate que foram adquiridos em um atacado apresentaram uma AR de 90mg CO $\mathrm{kg}^{-1} \mathrm{~h}^{-1}$, enquanto que frutos colhidos diretamente na planta apresentaram valores médios de $60 \mathrm{mg} \mathrm{CO} \mathrm{kg}^{-1} \mathrm{~h}^{-1}$. Durante o processo de limpeza, as cerdas mais flexíveis, náilon e sisal, combinadas às rotações de 80 e $120 \mathrm{rpm}$, envolviam os frutos, tornando o processo de limpeza mais eficiente, todavia aumentava a atividade respiratória na primeira hora, assim como foi observado para o aumento da perda de massa um dia após a limpeza (PELEG, 1985; BRECHT, 2002; PARISH et al., 2003). No caso das cerdas de fibra de coco combinada as rotações de 80 e 160rpm, observouse que os frutos apresentaram altos valores de AR, indicando que cerdas de maior rigidez influenciam o metabolismo dos frutos, independentemente da velocidade de funcionamento aplicada.

A evolução da concentração de etileno nos frascos variou $0,05 \mu \mathrm{L} \mathrm{C}_{2} \mathrm{H}_{4} \mathrm{~kg}^{-1} \mathrm{~h}^{-1}$ na primeira hora para os tratamentos fibra de coco 120 e 160rpm até $3,48 \mu \mathrm{L} \mathrm{C}_{2} \mathrm{H}_{4} \mathrm{~kg}^{-1} \mathrm{~h}^{-1}$ na quarta hora após o processo de

Tabela 2 - Valores médios e comparação de médias da atividade respiratória $\left(\mathrm{mg} \mathrm{CO}_{2} \mathrm{~kg}^{-1} \mathrm{~h}^{-1}\right)$ e concentração de etileno $\left(\mu \mathrm{L} \mathrm{kg}^{-1} \mathrm{~h}^{-1}\right) \mathrm{em}$ tomates 'Débora'. A atividade respiratória e a concentração de etileno foram obtidas para os frutos submetidos à limpeza nas escovas com cerdas de náilon, sisal e fibra de coco e nas rotações de 80, 120 e 160rpm, nas quatro horas de acompanhamento.

\begin{tabular}{|c|c|c|c|c|c|c|c|c|c|}
\hline \multirow{3}{*}{\multicolumn{2}{|c|}{ Tratamento }} & \multicolumn{4}{|c|}{------Atividade Respiratória $\left(\mathrm{mg} \mathrm{CO}_{2} \mathrm{~kg}^{-1} \mathrm{~h}^{-1}\right)$------ } & \multicolumn{4}{|c|}{---Concentração de etileno $\left(\mu \mathrm{L} \mathrm{kg}^{-1} \mathrm{~h}^{-1}\right)$} \\
\hline & & \multicolumn{8}{|c|}{ - } \\
\hline & & 1 & 2 & 3 & 4 & 1 & 2 & 3 & 4 \\
\hline \multirow[t]{2}{*}{ Controle } & & $54,80^{\mathrm{c}}$ & $50,75^{\mathrm{c}}$ & $49,67^{\mathrm{d}}$ & $48,33^{\mathrm{b}}$ & $0,04^{\mathrm{b}}$ & $0,06^{\mathrm{c}}$ & $0,23^{\mathrm{e}}$ & $0,38^{\mathrm{e}}$ \\
\hline & $80 \mathrm{rpm}$ & $67,24 \mathrm{a}^{\mathrm{c}}$ & $61,01^{\mathrm{abc}}$ & $57,62^{\mathrm{abcd}}$ & $54,76^{\mathrm{ab}}$ & $0,79^{\mathrm{a}}$ & $1,43^{\mathrm{a}}$ & $1,89^{\mathrm{ab}}$ & $2,66^{\mathrm{ab}}$ \\
\hline \multirow{3}{*}{ Náilon } & $120 \mathrm{rpm}$ & $74,90^{\mathrm{ab}}$ & $70,31^{\mathrm{a}}$ & $68,29^{\mathrm{a}}$ & $65,58^{\mathrm{a}}$ & $0,68^{\mathrm{ab}}$ & $1,54^{\mathrm{a}}$ & $1,90^{\mathrm{ab}}$ & $2,34^{\mathrm{bc}}$ \\
\hline & $160 \mathrm{rpm}$ & $56,45^{\mathrm{c}}$ & $55,35^{\mathrm{bc}}$ & $50,55^{\mathrm{cd}}$ & $48,88^{\mathrm{ab}}$ & $0,25^{\mathrm{ab}}$ & $0,90^{\mathrm{ab}}$ & $1,34^{\mathrm{bc}}$ & $2,48^{\mathrm{abc}}$ \\
\hline & $80 \mathrm{rpm}$ & $77,47^{\mathrm{a}}$ & $71,72^{\mathrm{a}}$ & $65,62^{\mathrm{ab}}$ & $59,56^{\mathrm{ab}}$ & $0,38^{\mathrm{ab}}$ & $0,87^{\mathrm{ab}}$ & $1,10^{\mathrm{cd}}$ & $1,57^{\mathrm{cd}}$ \\
\hline \multirow{3}{*}{ Sisal } & $120 \mathrm{rpm}$ & $70,80^{\mathrm{abc}}$ & $52,55^{\mathrm{c}}$ & $59,23^{\mathrm{abcd}}$ & $56,06^{\mathrm{ab}}$ & $0,29^{\mathrm{ab}}$ & $0,50^{\mathrm{bc}}$ & $1,33^{\mathrm{bc}}$ & $2,49^{\mathrm{abc}}$ \\
\hline & $160 \mathrm{rpm}$ & $59,65^{\mathrm{bc}}$ & $62,29^{\mathrm{abc}}$ & $56,83^{\mathrm{abcd}}$ & $55,28^{\mathrm{ab}}$ & $0,52^{\mathrm{ab}}$ & $0,73^{\mathrm{bc}}$ & $2,13^{\mathrm{a}}$ & $3,48^{\mathrm{a}}$ \\
\hline & $80 \mathrm{rpm}$ & $72,82^{\mathrm{ab}}$ & $67,74^{\mathrm{ab}}$ & $62,71^{\mathrm{abc}}$ & $58,30^{\mathrm{ab}}$ & $0,06^{\mathrm{b}}$ & $0,15^{\mathrm{bc}}$ & $0,28^{\mathrm{e}}$ & $0,41^{\mathrm{e}}$ \\
\hline \multirow[b]{2}{*}{ Fibra de Coco } & $120 \mathrm{rpm}$ & $58,62^{\mathrm{bc}}$ & $54,41^{\mathrm{bc}}$ & $54,29^{\mathrm{bcd}}$ & $53,02^{\mathrm{ab}}$ & $0,05^{\mathrm{b}}$ & $0,12^{\mathrm{c}}$ & $0,33^{\mathrm{e}}$ & $0,67^{\mathrm{de}}$ \\
\hline & $160 \mathrm{rpm}$ & $76,64^{\mathrm{a}}$ & $71,27^{\mathrm{a}}$ & $68,41^{\mathrm{a}}$ & $63,42^{\mathrm{ab}}$ & $0,05^{\mathrm{b}}$ & $0,23^{\mathrm{bc}}$ & $0,49^{\text {de }}$ & $0,62^{\mathrm{de}}$ \\
\hline
\end{tabular}

Médias com mesma letra na mesma coluna não diferem entre si pelo teste de Tukey, $\mathrm{P}<0,05$.

Ciência Rural, v.39, n.9, dez, 2009. 
limpeza no tratamento sisal 160rpm (Tabela 2). LEE et al. (2004) também observaram um aumento na concentração de etileno em frutos de tomate que foram colhidos no estádio verde maduro e sofreram danos físicos, ou seja, uma hora após o impacto, a produção de etileno era de 3 a $4 \mu \mathrm{L} \mathrm{C}_{2} \mathrm{H}_{4} \mathrm{~kg}^{-1} \mathrm{~h}^{-1}$ e após quatro horas estava entre 5 e $6,5 \mu \mathrm{L}_{2} \mathrm{C}_{4} \mathrm{~kg}^{-1} \mathrm{~h}^{-1}$. Neste trabalho, observou-se que frutos que passaram pelo processo de limpeza apresentaram maiores valores para a produção de etileno, quando comparados a frutos de controle. MACLEOD et al. (1976) e LEE et al. (2004) descrevem que, após uma hora de sofrerem injúrias, os frutos já apresentam um incremento na produção de etileno. Neste trabalho, uma maior evolução na concentração de etileno foi observada para os frutos submetidos à limpeza nos tratamentos náilon e sisal, indicando que esses tratamentos provocaram maior estresse nos frutos e consequentemente maiores alterações metabólicas.

\section{CONCLUSÕES}

As cerdas de náilon $0,15 \mathrm{~mm}$, por serem mais flexíveis e envolverem as esferas e os frutos, mostraramse mais eficientes no processo de limpeza que cerdas de menor flexibilidade (fibra de coco), em especial quando combinadas às rotações de 80 e 120rpm. Todavia, mais alterações foram observadas nos frutos quando essas rotações foram comparadas à rotação de $160 \mathrm{rpm}$. As cerdas do tipo PET, com maior rigidez, mostraram-se ineficientes no processo de limpeza das esferas, já as cerdas de fibra de coco, de menor flexibilidade que as de náilon, não demonstraram eficiência no processo de limpeza, mais especificamente, nos tratamentos de menor rotação. Portanto, no caso de um produto com baixa sujidade, deve-se levar em conta a flexibilidade da cerda, que proporcionará uma maior integração cerda/fruto.

\section{AGRADECIMENTOS}

À Coordenação de Aperfeiçoamento de Pessoal de Nível Superior (Capes), à Fundação de Amparo à Pesquisa do Estado de São Paulo (FAPESP), projeto 02/00645-7, e ao sistema PRODETAB/EMBRAPA, projeto 55-02/01.

\section{REFERÊNCIAS}

ANDRADE JÚNIOR, V.C. Avaliação do potencial produtivo e da firmeza pós-colheita de frutos em híbridos de tomateiro. 1999. 52f. Dissertação (Mestrado em Genética e Melhoramento de Plantas) - Universidade Federal de Lavras, Lavras, MG

ARTÉS, F.; ARTÉS-HERNÁNDEZ, F. Tratamientos postrecolección del tomate fresco: Tendencias e Innovaciones.
In: NAMESNY, .A. (Coord.) Tomates: producción y comercio. Reus (España): Ediciones de Horticultura, 2004. Cap.10, p.109120 .

BRECHT, J.K. Harvest and handling techniques. In: BARTZ, J.A. (ed). Postharvest physiology and pathology of vegetables. 2.ed. New York: Marcel Dekker, 2002. p.383392 .

CALEGARIO, F. et al. Determination of the respiration rate of tomato fruit using flow injection analysis. Postharvest Biology And Technology, v.22, p.249-256, 2001.

CHITARRA, M.I.F.; CHITARRA, A.B. Pós-colheita de frutas e hortaliças: fisiologia e manuseio. 2.ed. Lavras: UFLA, 2005. 785p

FERREIRA, M.D. et al. Avaliação da etapa da colheita em tomates de mesa cv. Débora. Brazilian Journal of Food Technology, v.7, n.2, p.173-178, 2004.

FERREIRA, M.D. et al. Avaliação física do tomate de mesa 'Romana' durante manuseio na pós-colheita. Engenharia Agrícola, v.26, n.1, p.321-327, 2006.

FNP Consultoria \& Comercio. AGRIANUAL 2007 - Anuário Estatístico da Agricultura Brasileira. 10.ed. São Paulo, 2006. p.495-502

FRANCO, A.T.O. et al. Padrões de funcionamento para equipamento nacional de beneficiamento e classificação para tomate de mesa. In: CONGRESSO BRASILEIRO DE ENGENHARIA AGRÍCOLA, 33., 2004, São Pedro. Anais... São Pedro: SBEA, 2004. (Cd-Rom).

FRANCO, A.T.O. et al. Avaliação do processo de limpeza em protótipo do sistema UNIMAC*. In: CONGRESSO BRASILEIRO DE ENGENHARIA AGRÍCOLA, 34., 2005, Canoas. Anais... Canoas: SBEA, 2005. (Cd-Rom).

HYDE, G.M.; ZHANG, W. Apple bruising research update: packingline impact evaluations. Tree Fruit Postharvest Journal, v.3, n.3, p.12-15, 1992.

KADER, A.A. Postharvest biology and technology: an overview. In: KADER, A.A. (Ed). Postharvest technology of horticultural crops. 2.ed. Davis: University of California, 1992. p.15-20.

KADER, A.A.; MORRIS L.L. Prompt handling reduces processing-tomato losses. California Agriculture, 1978. Disponível em: $<\mathrm{http} / /$ postharvest.ucdavis.edu/datastorefiles/ 234-279.pdf>. Acesso em 25 de Jun. de 2007. doi\#10.3733/ ca.

KADER, A.A.; SALTVEIT, M.E. Respiration and gas exchange. In: BARTZ, J.A.; BRECHT, J.K. (Eds.). Postharvest physiology and pathology of vegetables. New York: Marcel Dekker, 2003. p.7-29.

KAYS, S.J. Postharvest physiology of perishable plant products. New York: Van Nostrand Reinhold, 1991. 532p.

LANA, M.M.; FINGER, F.L. Atmosfera modificada e controlada. Aplicação na conservação de produtos hortícolas. Brasília: Embrapa Comunicação para Transferência de Tecnologia/Embrapa hortaliças, 2000. 34p. 
LEE, E. et al. Impact thresholds to maximize postharvest quality of Roma-type tomato. Proceedings of the Florida State Horticultural Society, v.117, p.373-377, 2004.

MACLEOD, R.F. et al. Stimulation of ethylene and $\mathrm{CO}_{2}$ production of mature-green tomatoes by impact bruising. HortScience. v.11, n.6, p.604-606, 1976.

MILLER, W.M. et al. Packingline machinery for Florida citrus packinghouses. Florida: Cooperative Extension Service, Institute of Food and Agricultural Sciences, University of Florida 2001. 26p. (Extension Bulletin 239). Disponível em: < http://edis.ifas.ufl.edu/BODY_AE184 >. Acesso 11 mar. 2005 .

MILLER, W.M.; ISMAIL, M.A. Injury assessment in citrus packing operations. Disponível em: < http://flcitrus.ifas.ufl.edu/ UF\% 20 IFAS $\% 20$ Short $\% 20$ Course \%20 Proceedings/ Quality\%20Control/quality55-64high.pdf > Acesso 11 mar 2005.

MOURA, M.L. et al. Fisiologia do amadurecimento na planta do tomate 'Santa Clara' e do mutante 'Firme'. Horticultura Brasileira, v.23, n.1, p.81-85, 2005.

NAIKA, S. et al. A cultura do tomate: produção, processamento e comercialização. Wageningen: Fundação Agromisa e CTA, 2006. 104p.
PARISH, M.E. et al. Methods to reduce/eliminate pathogens from fresh and fresh-cut produce. Comprehensive reviews in food science and food safety, v.2, p.161-173, 2003.

PELEG, K. Produce handling, packing and distribution. Westport: AVI, 1985. 625p.

RAWL, H.R. et al. A mathematical model for predicting toothbrush stiffness. Dental Materials, v.6, n.2, p. 111-117, 1990 .

SARGENT, S.A. et al. Assessment of mechanical damage in tomato packing lines. Transactions of the ASAE, v.30, n.1, p.630-634, 1989.

SIGRIST, J.M.M. et al. Manuseio pós-colheita de frutas e hortaliças. In: CORTEZ, L.A.B. et al. (Eds.). Resfriamento de frutas e hortaliças. Brasília: Embrapa Hortaliças, 2002. 428 p.

SILVA, M.C. et al. Avaliação da eficiência de limpeza em equipamentos de beneficiamento do tomate de mesa utilizandose sistema hidráulico alternativo. In: CONGRESSO BRASILEIRO DE ENGENHARIA AGRÍCOLA, 35., 2006, João Pessoa. Anais... João Pessoa: SBEA, 2006. (Cd-Rom).

VILELA, N.J. et al. O peso da perda de alimentos para a sociedade: o caso das hortaliças. Horticultura Brasileira, v.21, n.2, p. 141-143, 2003. 